major hazard in fission reactions, and it may be that chunk emission must be guarded against with equal care in the fusion reactors of the future.

\section{Cancer in Tuscany}

from R. A. Knight and N. A. Mitchison

BANNERS decked the streets of Florence, Sienna, Montecatini, Pisa and Lucca, for the 6,000 members who came to listen to papers given at the Eleventh International Cancer Congress by some 4,500 authors on October 20 26. Buses and trains shuffled to and fro between the five cities, but we found the delight of autumn in Tuscany compensated for the fuss. The congress accurately conveyed the flavour of cancer research over the past four years: greatly to oversimplify it was one of slow progress in cell biology and immunology, with dramatic news concerning viruses. We listened to the virology and immunology papers.

In the RNA tumour virus field, new work localised specific viral antigens on the tumour cell surface. D. P. Bolognesi (Duke University) showed that monospecific anti-p30 and antigp71 sera were cytotoxic for virus producer and non-producer methylcholarthene induced mouse tumours, although the anti-p30 effect may be mediated by antigen non-specifically absorbed to the cell surface. Natural mouse antibody is also cytotoxic for these cells, and can be partially absorbed both by gp71 and by $\mathrm{p} 30$. The predominant anti-viral antibodies in the serum of normal wild and laboratory strain mice, reported by R. C. Nowinski (University of Wisconsin), react with p15, and with both viral envelope glycoproteins. Nowinski also finally laid to rest the old dogma that mice cannot mount a humoral anti-p30 response, since this is the major specificity in the serum of C57BL mice immunised with the K36 leukaemia.

Several workers discussed the relationship of oncogenic viruses to human tumours. G. Klein (Karolinska Institute) reported that although $98 \%$ of African Burkitt lymphoma biopsies were positive both by nucleic acid hybridisation for EBV DNA, and for the EpsteinBarr nuclear antigen, European cases were negative for the genome. $\mathrm{S}$. Speigelman (Columbia University) summarised evidence from his laboratory for an association between 70S RNA and a reverse transcriptase-containing particle in a variety of human tumours, and showed that this agent contained a protein which cross reacts with p25 from the Mason-Pfizer agent. R. C. Gallo (National Institutes of Health) found that antisera to primate virus reverse transcriptase cross react with an antigen from human leukaemic cells, and that human tumour cells contain an antigen cross-reactive with primate virus p30. Visual sightings of the mature Ctype particles in human tissue material, however, are rare. Non-enveloped particles, which contain 70S RNA and viral antigens (virosomes), and which actively incorporate labelled precursors in vitro, were described in the mitochondrial membrane of avian and murine tumours by J. Kara (Czechoslovak Academy of Sciences, Prague). These particles are more prominent in nonproducer tumours. Although the Spiegelman particles, but not the oncornavirus precursors identified in human cell lines by A. Bukrinskaya (Academy of Medical Sciences, Moscow), differ from virosomes in density, more than one worker went home anxious to isolate human mitochondria. Thus immunology's most useful contribution to cancer research at present may be in the identification of candidate human tumour viruses.

Many papers dealt with the immune response to tumour-specific antigens. On the clinical side, the meaning of cell mediated immunity in vitro was questioned in several papers, for example by $M$. Takasugi (University of California, Los Angeles), R. W. Baldwin (University of Nottingham), and F. J. Cummings (Brown University): the popular microcytotoxicity test can no longer be accepted as a measure of the response of the patient to his own tumour. Significant responses, if they occur at all, seem to do so most clearly with a restricted range of tumours which include bladder carcinoma, melanoma and sarcomas. Encouraging results were reported with 'immunotherapy'-both specific (with administration of tumour cells or membrane preparations) and nonspecific (with immunological adjuvants such as tubercle bacilli, where C. Maruyama (University of Tokyo) had particularly extensive data). In no case, however, was there decisive evidence of the involvement of an immune mechanism.

The use of immunological methods to detect and monitor tumour growth was concentrated mainly on carcinoembryonic antigen for carcinoma of the gut and alpha-foetoprotein (AFP) for hepatoma, with limited use of cellular immunity against cell lines in other types of cancer. Papers from Europe and the United States, all of which took a cautious line towards widespread population screening were dwarfed by one from the Coordinating Group for AFP (Pekin) which included data from a massive screen of 500,000 individuals. Those who wish to evaluate the test wait, therefore, to see whether the Chinese find the effort worthwhile.

Analysis of the immunological mechanisms which operate in animal tumour systems continues. The entire response from induction to final killing can now be obtained in vitro, and the killer cells thus generated are highly active (J. C. Cerottini, Institute of Cancer Research, Lausanne). Efforts to characterise the antigens involved have now reached the stage reached 5-10 years ago with the major transplantation antigens. Virus-coded cell surface antigens are better characterised, and are available in some instances for in vitro manipulation with lymphocytes (G. R. Shellam, Imperial Cancer Research Fund, London). From the strictly immunological standpoint the furthest advance was reported by $\mathrm{K}$. Ratjewski (University of Koln), who has rendered antigens obsolete by elicitating a response with anti-idiotypic antibody, that is, with antibody directed against the antigen-combining region on the receptors of a clone of lymphoytes. Since his procedure stimulates $T$ lymphocytes as well as B lymphocytes, it implies-subject to certain safeguards in interpretationthat $T$ and $B$ cells read from a common dictionary and share a common pool of receptors.

\section{Tumour viruses at Munich}

\section{from a Correspondent}

Tumour virus meetings tend to have the same speakers, discuss the same subjects and send their participants away asking the same question: what do tumour viruses have to do with tumours? The clinical oncologist must helplessly face this question every day; meanwhile the basic oncologist faces the statistics that $60-90 \%$ of all known malignancies in man are caused by chemical carcinogens and that in no case so far have the candidate human cancer viruses been firmly identified as a cause of cancer in man. Their problems are intensified by the gap between experimental tumour research and clinical oncology. The tumour virus meeting held at the Max-Planck Institute for Biochemistry, Munich, from October 4 to 10 provided a good opportunity for communication between basic and clinical oncologists.

Dr R. Gallo (National Cancer Institute, Bethesda) discussed his concept of class I and class II viruses. He showed that the RNA genome of class $I$ viruses (typical endogenous viruses such RD-114) hybridises virtually completely to DNA of normal uninfected cells. These virogenes may be incompletly expressed and their products, such as reverse transcriptase, can be associated with normal tissues. Class II viruses show much less hybridisation of their RNA genome to uninfected cell DNA. Members of this class, for example murine and feline leukaemia 